\title{
openheart Effects of lockdown on acute coronary syndrome incidence in an area without community transmission of COVID-19
}

\author{
David Ferreira (D) , ${ }^{1}$ Simon Graffen, ${ }^{1}$ Brendan Watkins, ${ }^{1}$ Bridie Peters, ${ }^{1}$ \\ Geok Jim Lim, ${ }^{1}$ Harish Kamalanathan, ${ }^{1}$ James Leitch, ${ }^{1}$ Aaron Sverdlov, ${ }^{1,2}$ \\ Nicholas Collins, ${ }^{1,2}$ Andrew Boyle, ${ }^{1,2}$ Allan Davies ${ }^{1}$
}

To cite: Ferreira D, Graffen S, Watkins B, et al. Effects of lockdown on acute coronary syndrome incidence in an area without community transmission of COVID-19. Open Heart 2021;8:e001692. doi:10.1136/ openhrt-2021-001692

Received 16 April 2021 Accepted 1 June 2021

\section{Check for updates}

\section{(C) Author(s) (or their} employer(s)) 2021. Re-use permitted under CC BY-NC. No commercial re-use. See rights and permissions. Published by BMJ.

${ }^{1}$ Department of Cardiology, John Hunter Hospital, Newcastle, New South Wales, Australia

${ }^{2}$ Hunter Medical Research Institute, Newcastle, New South Wales, Australia

Correspondence to Dr David Ferreira; david. ferreira@health.nsw.gov.au

\section{ABSTRACT}

Objective To assess the changes in cardiac hospitalisations, acute coronary syndromes (ACS) and out-of-hospital cardiac arrest (OOHCA) during COVID-19 isolation compared with prior time periods in an area of low COVID-19 disease incidence.

Methods Review of all cardiology admissions, non-ST segment elevation myocardial infarction (NSTEMI), STsegment elevation myocardial infarction (STEMI) requiring urgent catheter laboratory activation and OOHCA. The 10-week period of government-imposed social isolation (23 March-31 May 2020) was compared with the same period in 2018, 2019 and a 10-week period prior to social isolation (6 January-15 March 2020). Incidence rate ratios were calculated. Symptom to balloon time was also compared for those requiring catheterisation laboratory activation for STEMI.

Results The incidence of COVID-19 in the health district was 0.14 per 100000 per day during the isolation period. There was a significant reduction in cardiology hospitalisations, NSTEMI and STEMI presentations without changes in OOHCA or symptom to balloon time for STEMI. Conclusions We observed a significant decline in cardiology presentations during social isolation without widespread COVID-19 disease. This provides further evidence for the important influence of social and behavioural factors on coronary event rates.

\section{INTRODUCTION}

During the COVID-19 pandemic, widespread use of social isolation has been instituted to reduce disease spread. A decrease in acute coronary syndrome (ACS) related hospital admissions was noted among populations with high rates of COVID-19. ${ }^{1}$ However, these results were significantly impacted by confounding due to high rates of COVID-19 transmission. During the mandatory lockdown period in the Hunter New England (HNE) region of Australia, with a total population of approximately 950000 , there was very low community transmission of COVID$19 .^{2}$ This has allowed the establishment of a natural control group to examine the true

\section{Key questions}

What is already known about this subject?

- The COVID-19 pandemic resulted in the international uptake of social isolation measures. Dramatic declines in acute coronary syndrome admissions were seen in areas of high COVID-19 incidence. The underlying mechanism behind this observation was unclear but was hypothesised to be related to patient reluctance to present to healthcare facilities.

What does this study add?

- This study demonstrates that social isolation measures were associated with dramatic declines in acute coronary syndrome admissions in the absence of widespread community COVID-19. This was not accompanied by changes in out-of-hospital cardiac arrest or changes in symptom to balloon time for ST-segment elevation myocardial infarction.

How might this impact on clinical practice?

- The decline in coronary event rates is remarkable and demonstrates the important influence of social and behavioural factors on cardiac presentations. This study does not appear to support the hypothesis that the decline in acute coronary syndrome presentations was solely due to patient reluctance to present to healthcare facilities.

effects of social isolation on ACS incidence rates, independent of the impact of widespread COVID-19 disease and transmission.

\section{METHODS}

We examined the rates of the following types of admissions: total cardiology, non-ST segment elevation myocardial infarction (NSTEMI), primary percutaneous coronary intervention (PCI) for ST segment elevation myocardial infarction (STEMI) and outof-hospital cardiac arrest (OOHCA) emergency department presentations to the John Hunter Hospital, the only tertiary hospital of the region. 
On 23 March 2020, chosen as the starting date, the Australian Federal Government officially enforced social isolation, announcing the closure of public food, entertainment and religious venues. On 31May, the chosen end date, the Australian Government relaxed restrictions, permitting local travel and reopening select public venues. The same dates were examined in 2018 and 2019. A 10-week pre-COVID-19 period was assessed between 6 January and 15 March 2020. Cardiology admissions, OOHCA and primary PCI records were sourced via the local patient administration system. NSTEMI presentations were collected from an ACS database that incorporated presentations across the entire district, including small community hospitals. This highly sensitive database identified all presentations with chest pain or elevated high-sensitivity troponin above the 99th centile. Patients in this dataset were excluded if they did not meet the Fourth Universal definition of type 1 myocardial infarction (MI). ${ }^{3}$ Population data were sourced from the Australian Bureau of Statistics. The population of the HNE district in 2018 was 927 607. Populations for 2019 and 2020 were extrapolated from 2018 with an average yearly increase of $1.26 \%$ in New South Wales over the past 10 years.

Normally distributed data were expressed as a mean with SD. Non-normally distributed data were expressed as a median with an IQR. Incidence rate ratios (IRRs) were calculated for each admission type comparing the COVID-19 isolation period to the prior time periods. The non-parametric continuous variable of symptom to balloon time was analysed with the Mann-Whitney U test.

\section{RESULTS}

The total number of new cases of COVID-19 in HNE during the isolation period was 96 , the majority of which were returned travellers. In a projected population of 951
129 , this reflects an incidence of 0.14 per 100000 per day. Of these, nine patients were hospitalised, one required intensive care and two died. This is over 30 times lower than the incidence experienced in Italy (4.3 per 100000 per day) during the period studied by De Filippo $e t a l^{45}$

Sixty-five per cent of presentations were male (table 1). There was a significant decline in total cardiology and NSTEMI admissions during the COVID-19 isolation period (see table 2). The number of ACS admissions per day was 4.4 in 2019 compared with 2.4 during COVID-19 isolation, which is a $45 \%$ reduction. The IRR of NSTEMI admissions during isolation compared with 2019 was 0.59 (95\% CI 0.47 to 0.73$)$, $\mathrm{p}$ value $<0.0001$. Primary catheterisation laboratory activation rates for STEMI were significantly lower during the COVID-19 isolation period, without any change in clinical practice guidelines (specifically, thrombolysis was not recommended to replace primary PCI in those with suspected COVID-19). The IRR of STEMI presentations for the isolation period compared with 2019 was 0.24 (95\% CI 0.14 to 0.40 ), p value $<0.0001$. This was not accompanied by a change in OOHCA presentations to the major tertiary centre of the region. There was no difference in symptom to balloon time for those with STEMI comparing prior time periods to COVID-19 isolation (see table 3).

\section{DISCUSSION}

In our unique population with exceptionally low incidence of COVID-19 infection, we observed lower rates of cardiology admissions, NSTEMI presentations and catheterisation laboratory activation for STEMI during the COVID-19 isolation period. This was not associated with changes in OOHCA presentations to the emergency department or symptom to balloon time for STEMI. In comparison, De Filippo et al found a similar $30 \%$

\begin{tabular}{lcccc}
\hline \multicolumn{4}{l}{ Table 1} & \multicolumn{4}{l}{ Baseline demographics and management of patients presenting with acute coronary syndrome based on time period } \\
\hline Characteristics & $\mathbf{2 0 1 8}$ & $\mathbf{2 0 1 9}$ & P-COV 2020 & COVID-19 2020 \\
\hline Total Ml numbers & 290 & 308 & 266 & 164 \\
\hline Age (SD) & $69.8( \pm 12.9)$ & $69.4( \pm 13.6)$ & $68.8( \pm 13.3)$ & $68.0( \pm 12.9)$ \\
\hline Males, \% (no.) & $65.9(191)$ & $65.6(202)$ & $60.9(162)$ & $69.5(114)$ \\
\hline Death at 28 days, \% (no.) & $4.5(13)$ & $7.1(22)$ & $6.0(16)$ & $3.0(5)$ \\
\hline NSTEMl, \% (no.) & $65.5(190)$ & $70.5(217)$ & $75.2(200)$ & $78.7(129)$ \\
\hline STEMI, \% (no.) & $34.5(100)$ & $29.5(91)$ & $24.8(66)$ & $21.3(35)$ \\
\hline P-PCl STEMI, \% (no.) & $25.9(75)$ & $23.0(71)$ & $18.0(48)$ & $10.4(17)$ \\
\hline Thrombolysed STEMl, \% (no.) & $8.6(25)$ & $6.5(20)$ & $6.8(18)$ & $11.0(18)$ \\
\hline Angiogram, \% (no.) & $78.6(228)$ & $78.6(242)$ & $73.3(195)$ & $74.4(122)$ \\
\hline Revascularisation, \% (no.) & $57.9(132)$ & $59.5(144)$ & $62.6(122)$ & $77.9(95)$ \\
\hline PCl, \% (no.) & $46.1(105)$ & $54.1(131)$ & $54.9(107)$ & $68.0(83)$ \\
\hline CABG, \% (no.) & $11.8(27)$ & $5.4(13)$ & $7.8(15)$ & $9.8(12)$
\end{tabular}

CABG, coronary artery bypass graft surgery; MI, myocardial infarction; NSTEMI, non-ST segment elevation myocardial infarction; OOHCA, out-of-hospital cardiac arrest; PCI, percutaneous coronary intervention; P-COV, pre-COVID-19 isolation; P-PCl, primary percutaneous coronary intervention; STEMI, ST-segment elevation myocardial infarction. 
Table 2 Comparison of cardiology admissions, NSTEMI admissions, primary catheterisation laboratory activation for STEMI and OOHCA presentations between COVID-19 isolation and three prior time periods

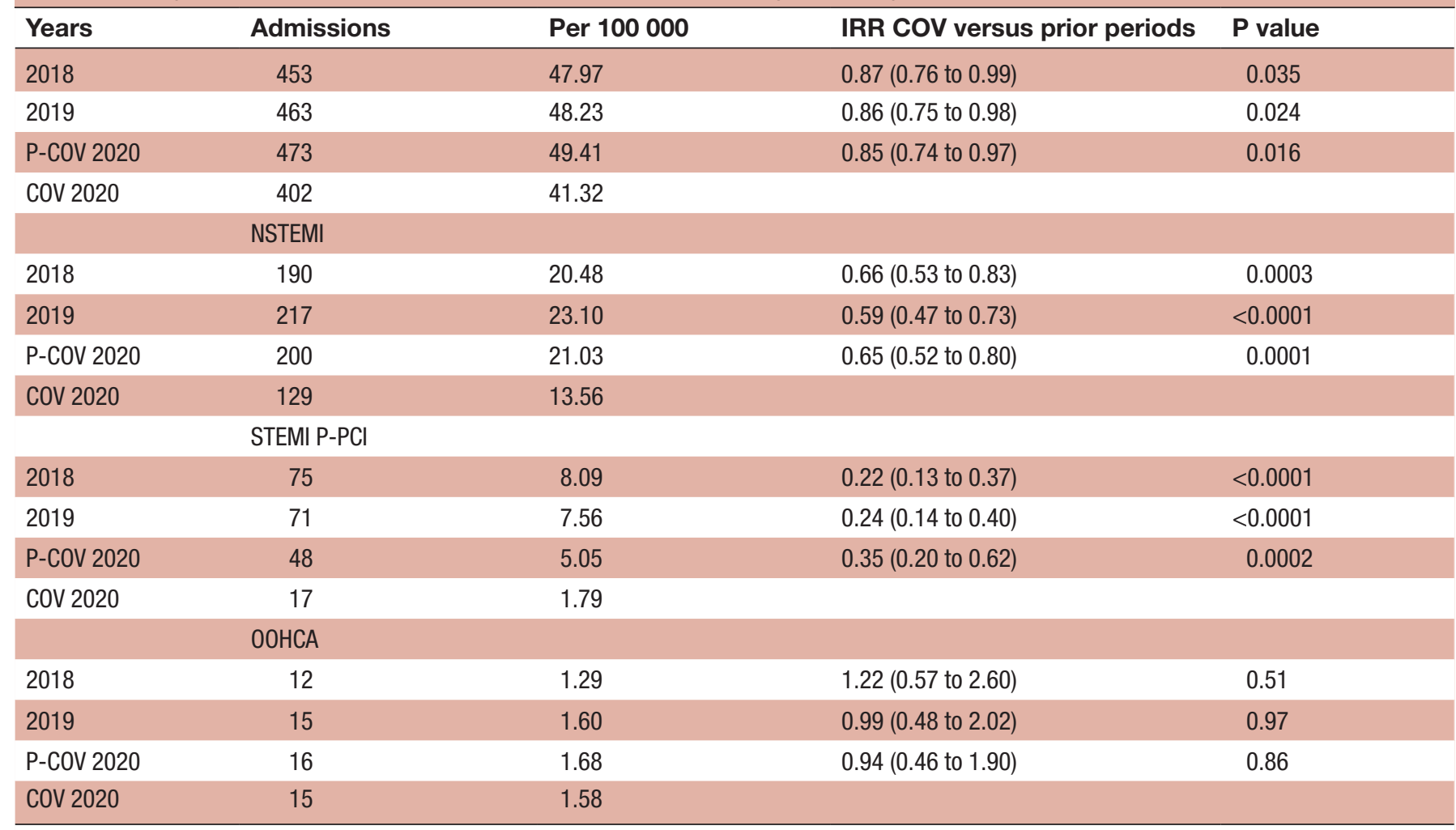

COV, COVID-19; IRR, incidence rate ratio; NSTEMI, non-ST segment elevation myocardial infarction; OOHCA, out-of-hospital cardiac arrest; P-COV, pre-COVID-19 isolation; P-PCI, primary percutaneous coronary intervention; STEMI, ST-segment elevation myocardial infarction.

reduction in ACS admissions per day, 18.9 in the prior year compared with 13.3 during COVID-19. ${ }^{4}$

There are several potential reasons for this observation in our population. There is a significant association between respiratory tract infections and rates of MI. ${ }^{6}$ Social isolation has resulted in a reduced incidence of respiratory infective illness in the community. ${ }^{7}$ This decreased inflammatory milieu may reduce risk of plaque destabilisation and MI. ${ }^{8}$ Second, while habitual exercise decreases cardiovascular risk, acute vigorous exercise can increase risk of ACS. ${ }^{9}$ Activity modification with reduced exercise during social isolation may have contributed to lower short-term rates of MI. Third, behavioural changes in the population due to fear of contracting COVID-19

Table 3 Comparison of symptom to balloon times for STEMI presentations using the Mann-Whitney $U$ test between COVID-19 isolation and three prior time periods

\begin{tabular}{lll}
\hline Years & $\begin{array}{l}\text { Symptom to } \\
\text { balloon time }\end{array}$ & $\begin{array}{l}\text { COV versus prior } \\
\text { periods ( } \mathbf{p} \text { value) }\end{array}$ \\
\hline 2018 & $366( \pm 334)^{\star}$ & 0.97 \\
2019 & $345( \pm 493)^{\star}$ & 0.83 \\
P-Cov 2020 & $332( \pm 239)^{\star}$ & 0.83 \\
COVID-19 2020 & $324( \pm 274)^{\star}$ & \\
\hline
\end{tabular}

${ }^{*} \mathrm{SD}$.

COV, COVID-19; P-Cov, pre-COVID-19 isolation. from healthcare facilities may have led to decreased hospital presentations. However, the most dramatic difference was on catheter laboratory activation for STEMI. This would be predicted to be the least affected, due to the typically more severe nature of symptoms, if patient reluctance were contributing to reductions in presentations.

Although there appeared to be a decrease in ACS incidence during the lockdown, behavioural changes observed in other studies may not be beneficial for longterm cardiovascular health. A study from Denmark found $28 \%$ of surveyed participants reported eating more during the isolation period. ${ }^{10}$ Sedentary behaviour also increased during social isolation in the UK. ${ }^{11}$ Others argue that lockdown from the COVID-19 pandemic may increase population risk of cardiovascular events long term. ${ }^{12}$ The degree to which isolation measures may have contributed to cardiac morbidity is unable to be answered based on this review.

Community OOHCA not presenting to the emergency department was not included, so our assessment of cardiac arrest incidence may be limited. One limitation is that the extent to which social isolation simply delayed the inevitable occurrence of a coronary event cannot be answered by this observational study. Nonetheless, the profound effect on the incidence of STEMI is remarkable and does illustrate the important 
influence of social and behavioural factors on coronary event rates.

Contributors DF: data collection, statistical analysis and authorship of the first and final draft. SG, BW, BP, GJL and HK: data collection, research design and statistical analysis. JL and AS: research design and editing the article from an epidemiological perspective. NC and AB: research design and editing the article from an acute coronary syndrome perspective. AD: senior author who proposed the research question, research design, assisted in authorship of the manuscript and performed statistical analysis.

Funding The authors have not declared a specific grant for this research from any funding agency in the public, commercial or not-for-profit sectors.

Competing interests None declared.

Patient consent for publication Not required.

Ethics approval This study was approved by the Hunter New England Human Research Ethics Committee.

Provenance and peer review Not commissioned; externally peer reviewed.

Data availability statement All data relevant to the study are included in the article.

Open access This is an open access article distributed in accordance with the Creative Commons Attribution Non Commercial (CC BY-NC 4.0) license, which permits others to distribute, remix, adapt, build upon this work non-commercially, and license their derivative works on different terms, provided the original work is properly cited, appropriate credit is given, any changes made indicated, and the use is non-commercial. See: http://creativecommons.org/licenses/by-nc/4.0/.

ORCID iD

David Ferreira http://orcid.org/0000-0003-3142-4503

\section{REFERENCES}

1 Solomon MD, McNulty EJ, Rana JS, et al. The Covid-19 pandemic and the incidence of acute myocardial infarction. N Engl J Med Overseas Ed 2020;383:691-3.

2 COVID-19 in NSW. New South Wales health, 2020. Available: https:// www.health.nsw.gov.au/Infectious/covid-19/Pages/recent-caseupdates.aspx

3 Thygesen K, Alpert JS, Jaffe AS, et al. Fourth universal definition of myocardial infarction (2018). Circulation 2018;138:e618-51.

4 De Filippo O, D'Ascenzo F, Angelini F, et al. Reduced rate of hospital admissions for ACS during Covid-19 outbreak in northern Italy. $N$ Engl J Med 2020;383:88-9.

5 Italy: coronavirus cases, 2020. Available: https://www.worldometers. info/coronavirus/country/italy/ [Accessed 2020].

6 Barnes M, Heywood AE, Mahimbo A, et al. Acute myocardial infarction and influenza: a meta-analysis of case-control studies. Heart 2015;101:1738-47.

7 Nascimento MS, Baggio DM, Fascina LP, et al. Impact of social isolation due to COVID-19 on the seasonality of pediatric respiratory diseases. PLoS One 2020;15:e0243694.

8 Granger $\mathrm{CB}$, Kochar A. Understanding and targeting Inflammation in acute Myocardial infarction: an elusive goal. J Am Coll Cardiol 2018;72:199-201.

9 Thompson Paul D, Franklin Barry A, Balady Gary J. Exercise and acute cardiovascular events. Circulation 2007;115:2358-68.

10 Giacalone D, Frøst MB, Rodríguez-Pérez C. Reported changes in dietary habits during the COVID-19 Lockdown in the Danish population: the Danish COVIDiet study. Front Nutr 2020;7:592112.

11 Spence JC, Rhodes RE, McCurdy A, et al. Determinants of physical activity among adults in the United Kingdom during the COVID-19 pandemic: the DUK-COVID study. Br J Health Psychol 2021;26:588-605.

12 Peçanha T, Goessler KF, Roschel $\mathrm{H}$, et al. Social isolation during the COVID-19 pandemic can increase physical inactivity and the global burden of cardiovascular disease. Am J Physiol Heart Circ Physiol 2020;318:H1441-6. 